\title{
Der Arzt als Patient - ein Erfahrungsbericht
}

\section{Max Schreier}

Dr. med., Facharzt für Allgemeine Innere Medizin, Mitglied FMH

Als praktizierender Arzt erlebt man im Alltag eine eindeutige Rollenverteilung: hier der Arzt als Diagnostiker und Therapeut, dort der behandelte Patient. Sobald der Arzt selbst erkrankt oder verunfallt, findet ein Rollentausch statt: Der Arzt wird zum Patienten.

Während meiner 29-jährigen Praxis-Tätigkeit erlebte ich drei stationäre Spitalaufenthalte, seit der Praxisübergabe im Jahre 2002 kamen weitere vier Hospitalisationen hinzu. Im Folgenden möchte ich (mit Jahrgang 1938) aus ärztlicher Sicht über zwei stationäre Behandlungen berichten.

\section{Der Velounfall}

Am 22. Juni 2015 erlitt ich mit dem Rennvelo einen Unfall. Ich erkannte noch das Hinterrad des LKW und ver- nahm einen Knall, dann tauchte ich ab ins Nirwana. Als ich wieder zu mir kam, kümmerte sich ein Helfer um mich und wollte eine Ambulanz kommen lassen. Wie ich nachträglich erfuhr, habe ich abgelehnt mit den Worten: «Das ist nicht nötig, ich bin selbst Arzt.» Er erkannte jedoch meine desolate Situation und alarmierte Sanität und Polizei. Obwohl ich völlig desorientiert war, konnte ich ihm unsere Telefonnummer angeben. Der Velohelm erlitt Totalschaden, er hatte mich aber vor äusseren Kopfverletzungen bewahrt. Da sich der Unfall im Bernbiet ereignete, landete ich in einem

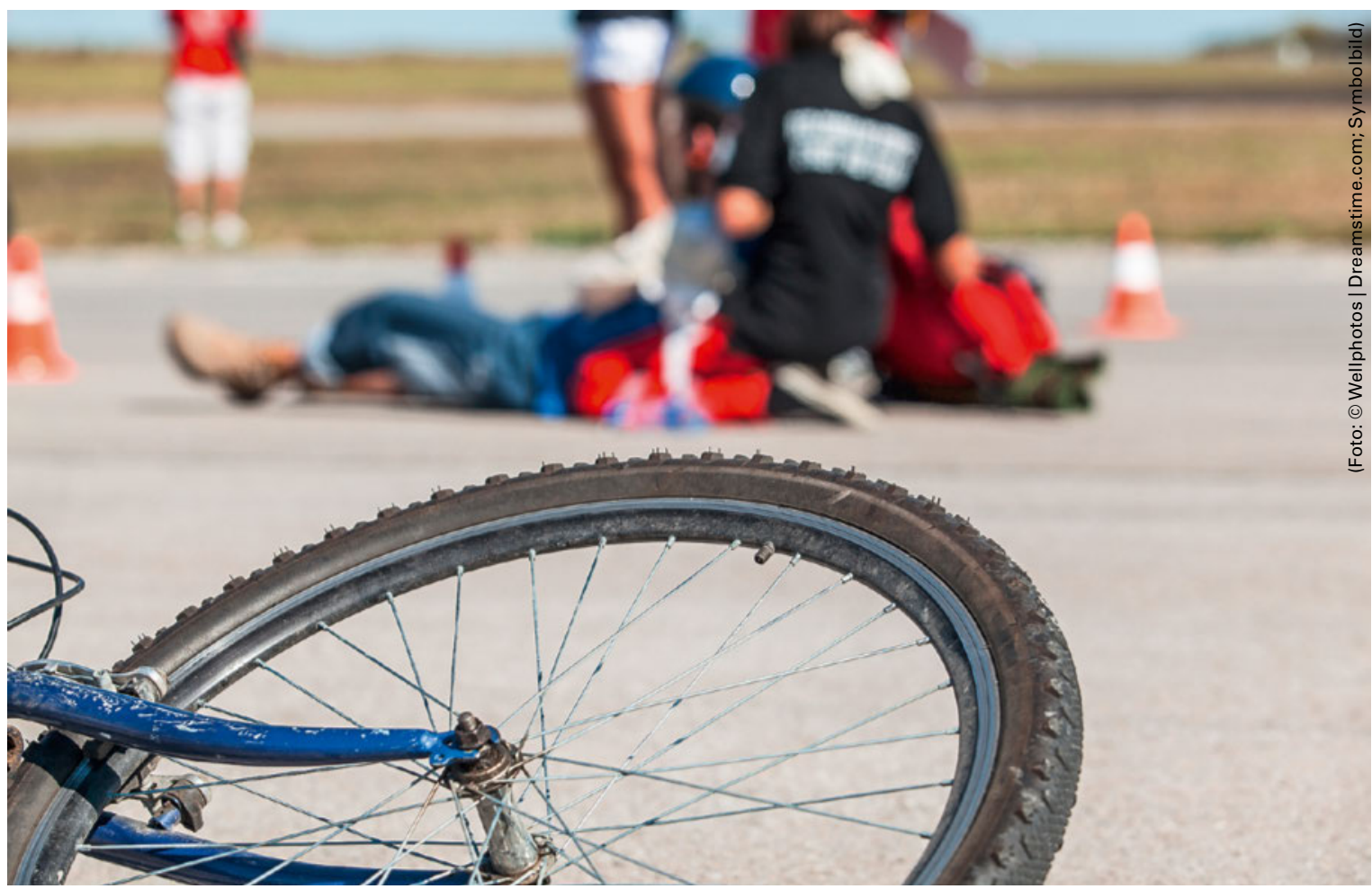

Max Schreier, Facharzt für Allgemeine Innere Medizin, erlitt mit seinem Velo einen Unfall und erkannte den Ernst der Lage selbst nicht. Er wollte einen Ersthelfer sogar davon abhalten, eine Ambulanz zu rufen. Zum Glück hat der Helfer nicht auf ihn gehört .. 
Regionalspital. Während der Fahrt mit der Ambulanz konnte ich meine retrograde Amnesie etwas einengen, indem ich die gefahrene Route nach anfänglicher totaler Desorientierung wieder aus dem Gedächtnis abrufen konnte.

Während fünf Stunden wurde ich auf der Notfallstation beobachtet und wiederholt getestet. Die leichten Kopfschmerzen und die Übelkeit wurden als Commotio cerebri interpretiert. Obwohl man mich noch eine Nacht behalten wollte, drängte ich nach Hause und

\section{Obwohl man mich noch eine Nacht behalten wollte, drängte ich nach Hause und wurde schliesslich entlassen.}

wurde schliesslich entlassen. Ein Polizist gab mir zu verstehen, dass ich den Unfall allein verschuldet habe und wegen Nichtbeherrschen des Fahrzeuges von der Staatsanwaltschaft des Kantons Bern noch eine Busse zu gewärtigen habe (acht Monate später erhielt ich den Strafbefehl mit einem Betrag von Fr. 200.-).

Anlässlich eines geplanten Arzttermins nach zwei Tagen wurde doch noch ein Schädel-CT durchgeführt, das angeblich keine Pathologien zeigte. Während einer Woche litt ich noch an Übelkeit und erträglichen Kopfschmerzen mit dem Vorteil, dass ich in dieser Zeit zwei Kilogramm abgenommen habe.

\section{Die Folgen}

Nach einem schmerzfreien Intervall stellten sich zwei Monate nach dem Unfallereignis besonders nachts wieder Kopfschmerzen ein. Bei einem Hundespaziergang fiel meiner Frau auf, dass ich das rechte Bein zeitweise auf dem Boden nachschleifte, ausserdem sei meine Sprache etwas verwaschen gewesen. Sie besorgte am 18. August einen Termin beim Hausarzt. Obwohl keine neurologischen Ausfälle nachweisbar waren, veranlasste er eine MRI-Untersuchung am folgenden Tag. Diagnose des Radiologen: chronisch subdurales ausgedehntes Hämatom links, schmales Hämatom rechts. In der Nacht vor dieser Untersuchung litt ich an starken Kopfschmerzen. Mit der Ambulanz wurde ich vom Regionalspital in die Neurochirurgische Klinik des Universitätsspitals überführt, wo wir um $17 \mathrm{Uhr}$ eintrafen. Nun ging das Warten los. Ich realisierte bald, dass die Neurochirurgen an diesem Abend am Laufband und nach Dringlichkeit operierten. Was mir aber Mühe bereitete, war die mangelnde Information. Erst als ich um 23 Uhr etwas energischer nachfragte, teilte man mir mit, dass ich morgens um zwei Uhr operiert werde. Durch zwei Bohrlöcher wurde das Hämatom in Allgemeinanästhe- sie entfernt. Während des postoperativen Aufenthaltes im Überwachungsraum wurde ich intensiv überwacht. Nach problemlosem Verlauf wurde ich in ein Doppelzimmer verlegt. Die ersten Tage verbrachte ich die meiste Zeit schlafend im Bett und musste vom Pflegepersonal immer wieder zur Tabletteneinnahme oder zum Essen geweckt werden. Ausserdem wurde auch während der Nacht der Blutdruck gemessen und der neurologische Status überprüft. Mir ist aufgefallen, dass diese Spezialklinik sehr personalintensiv betrieben wird. Es verging einige Zeit, bis ich als Kollege wahrgenommen wurde. Wenn ich einen Fachausdruck verwendete, wurde ich gefragt: «Sind Sie vom Fach?»

\section{Effizienter Spitalbetrieb}

Die morgendlichen Arztvisiten waren eindrücklich. Nach kurzem Klopfen an der Zimmertür rauschten etwa zehn Personen ins Zimmer mit drei Neurochirurgen, ein Arzt schob einen fahrbaren Untersatz mit aufgebautem Tablet-PC vor sich her. Die postoperativen Schmerzen konnten durch regelmässige perorale Einnahme von Analgetika meistens beherrscht werden. In einer Nacht hingegen war dies nicht ausreichend und die diensthabende Pflegefachfrau verabreichte mir eine Injektion mit Morphin. Soweit ich mich erinnere, war dies in meinem Leben die erste Morphindosis, die ich erhielt. Nach zwei Tagen konnte die Hirndrainage gezogen werden. Die postoperative Betreuung war vorbildlich. Dies habe ich im obligaten Fragebogen festgehalten. Die Entlassung erfolgte am fünften postoperativen Tag. Beim Austrittsgespräch erfuhr ich, dass in

\section{Nach einem schmerzfreien Intervall stellten sich zwei Monate nach dem Unfallereignis besonders nachts wieder Kopfschmerzen ein.}

zehn Prozent eine Nachblutung eintreten könne. Ich rechnete natürlich damit, dass ich zu jenen 90 Prozent gehöre, die ungeschoren davonkommen.

Wieder zu Hause, erhielt ich viele teilnehmende und aufmunternde Reaktionen. Stellvertretend möchte ich ein Mail eines pensionierten Solothurner Kollegen und Freundes, dessen Fortbildungskränzchen ich häufig besuchte, erwähnen: «Wir können Dir nur von Herzen weiterhin gute Besserung wünschen. Stelle fürderhin die Aufmerksamkeit Deiner Schutzengel nicht allzu sehr auf die Probe; auch sie werden älter, machen vielleicht ab und zu ein Nickerchen, oder sie feiern wie weiland während meiner Assistentenzeit die Klosterfrauen im Kreisspital Muri - die Vesper (während welcher letztere nicht gestört zu werden wünschten).» Am 24. September chauffierte mich meine Frau zur 
vorgesehenen Kontrolluntersuchung ins Inselspital. Nach dem Schädel-CT fand die Besprechung beim Neurochirurgen statt. Am Bildschirm war eine grosse Rezidivblutung mit Verschiebung der Seitenventrikel um sechs Millimeter zu erkennen. Wir waren überrascht über die Diskrepanz meiner geringen subjektiven Be-

\section{Wir waren überrascht über die Diskrepanz meiner geringen subjektiven Beschwerden und dem deutlichen Befund.}

schwerden und dem deutlichen Befund. Wir einigten uns auf die Reoperation am folgenden Tag. Am frühen Nachmittag fand die Reoperation statt.

Noch während der Nacht wurde ich in ein ZweibettZimmer verlegt. Ich döste vor mich hin und konnte meinen Bettnachbarn erst nach Stunden ansprechen. Wie ich erfuhr, hatte den 50-Jährigen ein ähnliches Schicksal ereilt: Velosturz auf dem Weg zur Arbeit, Entfernung eines subduralen Hämatoms durch zwei Bohrlöcher, Rezidivblutung nach vier Wochen.

Am ersten Tag nach der Operation wurde die Drainage entfernt und über beide Bohrlöcher ein Tupfer festgenäht. Bei unproblematischem Verlauf wurde nach vier Tagen mein Austritt beschlossen. Beim Austrittsgespräch mit zwei Neurochirurgen war meine Frau auch dabei. Bezüglich Prognose erhielten wir den überraschenden Bescheid, dass es nach einer derartigen Blutung in 20-30\% wieder bluten könne. Der einzige Trost: Mit zunehmender Dauer seit dem letzten Ereignis sinkt die Wahrscheinlichkeit einer erneuten Blutung. Sechs Wochen nach dem zweiten Eingriff fand die Schlusskontrolle statt. Die Befragung und neurologische Untersuchung (ohne erneutes CT) fiel sehr zufriedenstellend aus, so dass das Dossier geschlossen werden konnte.

\section{Persönliche Eindrücke eines Arztes}

Nach diesen stationären Behandlungen möchte ich folgende persönliche Eindrücke festhalten:

Korrespondenz: Dr. med. Max Schreier Arzt für Allgemeine Innere Medizin

Haltenstrasse 23

CH-4566 Kriegstetten schreier.max[at]bluewin.ch
1. Der Beruf als Arzt bietet keine Gewähr, dass man im Fall eines Unfalles oder einer akuten Erkrankung die Situation richtig einschätzt und die notwendigen und richtigen Entscheidungen trifft. Ich habe es den involvierten Helfern und besonders meiner Frau zu verdanken, dass ich die adäquate Hilfe erfahren durfte.

2. Die Diskrepanz zwischen subjektiven Beschwerden und objektivem Befund hat mich überrascht. Anlässlich der ausgedehnten Rezidivblutung verspürte ich lediglich eine gewisse Standunsicherheit. Die neurologischen Tests waren immer normal. Trotz unauffälligen klinischen Befunden sollten daher im Zweifelsfall apparative Untersuchungen beansprucht werden, die zur Diagnose führen können.

3. Die Kommunikation vor dem ersten Eingriff im Inselspital verlief nicht optimal. Probleme in der Kommunikation zwischen Arzt und Patient hatte ich auch als Ombudsmann der kantonalen Ärztegesellschaft öfters erlebt. Es lag mir fern, als Kollege eine Spezialbehandlung anzustreben. Wenn ich aber bei anderen ärztlichen Kontakten als Berufskollege wahrgenommen wurde, war die Verständigung erleichtert und meine Anliegen wurden ernster genommen und diskutiert. Ich bin mir jedoch bewusst, dass die Gefahr der Anonymität in einem grösseren und lebhafteren Klinikbetrieb steigt.

Beim Austrittsgespräch erfuhr ich, dass in zehn Prozent eine Nachblutung eintreten könne.

4. Die ärztliche Leistung und intensive pflegerische Betreuung habe ich hochqualifiziert erlebt. Diese Feststellung sollte man immer bedenken, wenn man über die hohen Kosten des helvetischen Gesundheitswesens debattiert.

Diese abschliessenden Bemerkungen möchte ich als aufbauende Kritik mit dem Wunsch nach Optimierung der Patientenbetreuung verstanden wissen. 